\title{
MPL W515 L/K mutations in myeloproliferative neoplasms
}

\author{
Sohaila Eldeweny ${ }^{1 *}$ (D) Hosny Ibrahim ${ }^{1}$, Ghada Elsayed ${ }^{2}$ and Mohamed Samra ${ }^{3}$
}

\begin{abstract}
Background: Myeloproliferative neoplasms (MPNs) describe a group of diseases involving the bone marrow (BM). Classical MPNs are classified into chronic myelogenous leukemia (CML), polycythemia vera (PV), essential thrombocythemia (ET), and primary myelofibrosis (PMF). This classification is based on the presence of Philadelphia (Ph) chromosome (BCR/ABL1). CML is BCR/ABL1-positive while PV, ET, and PMF are negative. JAK2 p. Val617Phe pathological variant is the most associated mutation in BCR/ABL1-negative MPNs. The frequency of JAK2 $\mathrm{p}$. Val617Phe is $90-95 \%$ in PV patients, $50-60 \%$ in ET, and $40-50 \%$ in patients with PMF. Studies on MPL gene led to the revelation of a gain of function pathological variants in JAK2 p. Val617Phe-negative myeloproliferative neoplasms (MPNs). MPL p. W515 L/K pathological variants are the most common across all mutations in MPL gene. The prevalence of these pathological variants over the Egyptian population is not clear enough. In the present study, we aimed to investigate the prevalence of MPL p. W515 L/K pathological variants in the Philadelphia (Ph)negative MPNs over the Egyptian population.
\end{abstract}

Results: We have tested 60 patients with Ph-negative MPNs for MPL p. W515 L/K pathological variants. Median age was 51 (22-73) years. No MPL p. W515 L/K pathological variants were detected among our patients. JAK2 p. Val617Phe in PV and PMF patients showed significantly lower frequency than other studies. Splenomegaly was significantly higher in ET patients compared to other studies.

Conclusion: MPL p. W515 L/K pathological variants are rare across the Egyptian Ph-negative MPNs, and further studies on a large number are recommended. MPN patients in Egypt are younger compared to different ethnic groups.

Keywords: MPL p. W515 L/K pathological variants, Myeloproliferative neoplasms, JAK2 p. Val617Phe, Polycythemia vera (PV), Essential thrombocythemia (ET), Primary myelofibrosis (PMF)

\section{Background}

Myeloproliferative neoplasms (MPNs) describe a group of diseases involving the bone marrow where there is a myeloid cell lineage proliferation that leads to an increase in the numbers of erythrocytes, megakaryocytes, or granulocytes in the bloodstream [1] Classical MPNs include chronic myelogenous leukemia (CML), polycythemia vera (PV), essential thrombocythemia (ET), and primary myelofibrosis (PMF) according to the World Health Organization (WHO) 2008 classification and the 2016 revision [2, 3]. Classical MPNs are classified according to the presence of Ph-chromosome (BCR/

\footnotetext{
* Correspondence: sohailaahmed28@hotmail.com

'Department of Chemistry, Faculty of Science, Cairo University, Al Gamaa street, Al Orman, Giza 12613, Egypt

Full list of author information is available at the end of the article
}

ABL1), CML is BCR/ABL1-positive while BCR/ABL1negative MPNs include PV, ET, and PMF [4].

Somatic pathological variant in exon 14 of the JAK2 gene $\mathrm{p}$. Val617Phe is the most associated mutation in BCR/ABL1-negative MPNs. The frequency of JAK2 $\mathrm{p}$. Val617Phe is $90-95 \%$ of patients meeting the clinical criteria for PV, $50-60 \%$ for $\mathrm{ET}$, and $40-50 \%$ for PMF [5]. While JAK2 exon 14 mutation is the most frequent mutation in MPN, about $10 \%$ of patients with clinical characteristics of PV, ET, and PMF lack this mutation [5]. Somatic variants in exon 12 of JAK2 gene are found exclusively in PV patients and are detected in one third of PV patients who are p. Val617Phe negative [6].

Pathological variants causing gain of function in the $M P L$ gene (thrombopoietin receptor) are detected in 
JAK2-negative ET and PMF patients. The most prominent $M P L$ pathological variants are located at the transmembrane region of the thrombopoietin receptor (TPO) [7-10].

The most frequent variants are found at codon 515 of the MPL gene being either p. Trp515Lys or p.Trp515Leu which is commonly referred to as MPL p. W515 L/K. The tryptophan residue (W) at position 515 in the MPL gene is responsible for the inhibition of the MPL transmembrane helix dimerization thus preventing the thrombopoietin-independent activation. The substitution of this residue with another amino acid such as leucine or lysine constitutively activates the JAK-STAT signaling and causes the thrombopoietin receptor to have uncontrolled self-activity leading to MPN [1, 10-13]. MPL p. W515 L/K variants are not found in PV patients but restricted to $3-5 \%$ in ET and 5-10\% in PMF [14].

The prevalence of these mutations over the Egyptian population still is not clear enough. In the present study, we aimed to investigate the frequency of MPL p. W515 L/K pathological variants in Philadelphia (Ph)-negative MPNs over the Egyptian population.

\section{Methods}

\section{Patients}

A total of 60 patients with MPNs were recruited for this study. All patients were referred to the outpatient clinic of Nasser institute hospital Cairo, Egypt. The study was carried out between February 2016 and January 2018. The diagnosis of MPN was based on the World Health Organization criteria (2016 revision) [3]. All patients were subjected to full clinical examination and laboratory studies. Patients with positive BCR/ABL were excluded. All patients gave informed consent, and the study was carried out per The Code of Ethics of the World Medical Association (Declaration of Helsinki) for experiments in humans.

\section{Treatment}

Low-dose aspirin was given to all PV and ET patients. For PV patients, repeated venesection was done to maintain hematocrit level below 45. Patients with PV/ ET, older than 60 years, and/or with previous history of thrombosis were considered as high risk and were given cytoreductive therapy with hydroxyurea. PMF patients with anemia were treated with repeated blood transfusion, danazol, erythropoietin, and corticosteroids whereas patients with huge splenomegaly were managed by hydroxyurea.

\section{Methods}

\section{DNA isolation}

Peripheral blood samples were collected at the time of diagnosis on EDTA anticoagulant tubes. Genomic DNA was extracted using QIAamp DNA Blood Mini Kit (Qiagen, Germany) (Cat: 51104) according to the manufacturer's instructions. DNA quality and quantity were assessed using Nanodrop.

\section{MPL p. W515 L/K pathological variants analysis}

Amplification refractory mutation system (ARMS) PCR technique was used for the screening of $M P L$ W515K/L pathological variants NM_005373.2: (p.Trp515Lys)/(p. Trp515Leu). ARMS PCR assay contains four primers (Table 1) in a single PCR tube, two forward and two reverse that are used in different combinations to amplify three probabilities, the internal control, wild allele, and the mutant allele bands. PCR was performed in $25 \mu \mathrm{l}$ reaction volume using one unit hot start Taq DNA (Cat: 154013297) as previously described by Zhuge et al. 2010 [15]. PCR products were evaluated on 1.5\% agarose gel (Fig. 1).

\section{JAK2 p. Val617Phe mutation analysis}

JAK2 p. Val617Phe mutation (NM_004972.3) c.1849G $>$ T (p. Val617Phe) was screened using ARMS technique. Two forward primers and two reverse primers were used (Table 2) in a single PCR tube to amplify three amplicons, an internal control, a wild allele, and mutant allele bands. PCR reaction contained one unit of Hot start Taq DNA polymerase (Cat: 154013297) as previously described by Chen et al. 2007 [16]. PCR products were analyzed on $2 \%$ agarose gel (Fig. 2).

\section{Statistical analysis}

Data were analyzed using SPSS windows statistical package, version 22 .

Table 1 Primers sequences for MPL p. W515 L/K pathological variants detection by ARMS technique

\begin{tabular}{ll}
\hline Primer & Sequence \\
\hline FO (forward outer) & 5'-GCCTGGATCTCCTTGGTGAC-3' \\
RO (reverse outer) & 5'-GAGGTGACGTGCAGGAAGTG-3' \\
Rwt (reverse wild type) & 5'-CTGTAGTGTGCAGGAAACTGtCA-3' \\
FL (forward mutant L) & 5'-GCCTGCTGCTGCTGAGaTT-3' \\
FK (forward mutant K) & 5'-GCCTGCTGCTGCTGAGtAA-3' \\
\hline
\end{tabular}




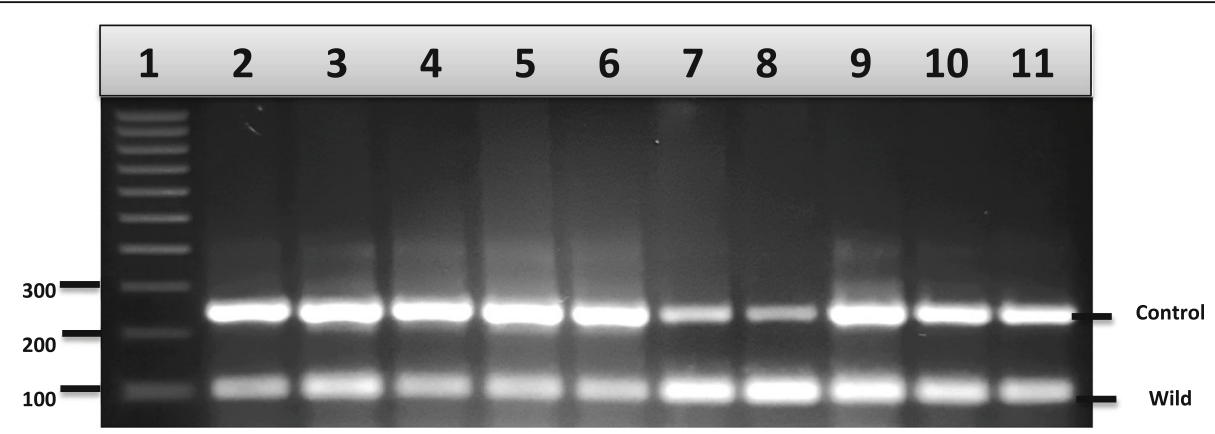

Fig. 1 Electrophoresis of amplified PCR products to detect MPL p. W515 L/K pathological variants by ARMS technique. Left to right: lane 1 is $100-$ base pair (bp) DNA ladder and lanes 2, 3, 4, 5, 6, 7, 8, 9, 10, and 11 are wild type cases showing only internal control band of 246 bp and wild type band of $98 \mathrm{bp}$

Numeric data were presented as mean and standard deviation or median and range as appropriate. Categorical variables were presented as frequency and percentage. Comparison between the different results regarding categorical data was done using chi-square test (Fisher's exact test). $P$ value less than 0.05 was considered significant, and all tests were two-tailed.

\section{Results}

The present study included 60 MPN patients: 14 (23.3\%) were diagnosed as PV, $26(43.3 \%)$ as ET, and $20(33.3 \%)$ as PMF. The median age was $51(22-73)$ years. No significant difference was detected between the three groups in age or sex, Table 1

\section{Laboratory characteristics}

Median WBC was $7.7(1.08-69) \times 10^{9} / \mathrm{L}$, Median hemoglobin was $12.9(3.1-26.3) \mathrm{g} / \mathrm{dL}$ and median platelet count was $497.5(13-2433) \times 10^{9} / \mathrm{L}$. Patients diagnosed with PV had significantly higher hemoglobin levels compared to ET and PMF (17.4 \pm 2.39 vs. $13 \pm 3.49 ; 8.7 \pm 2.8, p<0.001) \mathrm{g} / \mathrm{dl}$, respectively. Patients diagnosed with ET had a significantly higher level of platelet count compared to PV and PMF $(1099 \pm 470$ vs. $294 \pm 165,268.45 \pm 351.8, p<$ $0.001) \times 10^{9} / \mathrm{L}$, Table 3 .

Table 2 Primers sequences for JAK2 p.V617F mutation detection by ARMS technique

\begin{tabular}{ll}
\hline Primer & Sequence \\
\hline FO (forward outer) & 5'-TCCTCAGAACGTTGATGGCAG-3' \\
RO (reverse outer) & 5' ATTGCTTTCCTTTTCACAAGAT-3' \\
Fwt (forward wild type) & 5'-GCATTTGTTTAAATTATGGAGTATaTG-3' \\
Rmt (reverse mutant) & 5' GTTTACTTACTCTCGTCTCCACAaAA-3' \\
\hline
\end{tabular}

MPL p. W515 L/K pathological variants

All patients were negative for both pathological variants of the MPL W515 L and W515K

\section{JAK2 p. Val617Phe pathological variant}

$J A K 2$ p. Val617Phe mutation was detected in 28 (46.7\%) patients with no significant difference between the three groups, $p=0.143$. JAK2 p. Val617Phe pathological variant tended to be associated with the level of hemoglobin in patients with PV compared to ET and PMF $(16.689 \pm 2.4927$ vs. $12.838 \pm 2.4808$, $8.783 \pm 3.6581, p=0.062)$.

\section{Symptoms}

Fatigue was significantly higher in patients with PMF compared to those with PV and ET (12 (60\%), 5 (35.7\%), and $6(23.1 \%), p=0.04)$, respectively. Headache and dizziness were not significantly different between groups.

\section{Signs}

Splenomegaly was in $27 / 60$, (45\%) of patients. No significant difference was detected between groups regarding hepatomegaly or splenomegaly, Table 1.

\section{Complications}

Eleven (18.3\%) MPN patients had bleeding events; bleeding and thrombotic events did not show any significant difference between the three groups, Table 1 .

\section{Discussion}

In this study, we investigated the frequency of the pathological variant $M P L \mathrm{p}$. W $515 \mathrm{~L} / \mathrm{K}$ in 60 patients with Ph-negative MPNs. No MPL p. W515 L/K pathological variants were detected in our patients. Our results are consistent with previous studies performed among both Taiwanese and Iranian populations $[17,18]$. They screened 88 Taiwanese and 60 


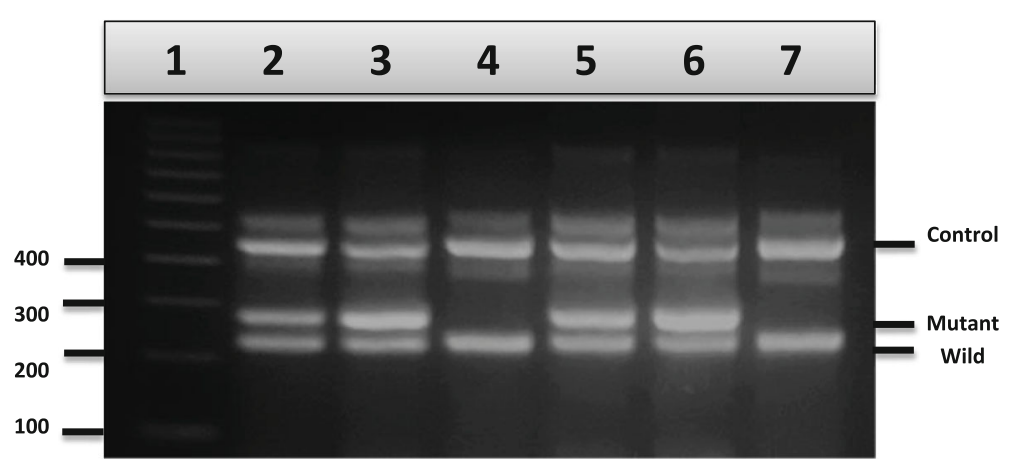

Fig. 2 Electrophoresis of amplified PCR products to detect JAK2 p. Val617Phe pathological variant in MPNs by ARMS technique. ARMS PCR shows an internal control band at $463 \mathrm{bp}$; the 229-bp band indicates the presence of the wild allele and the 279-bp band indicates the presence of the JAK2 p. Val617Phe mutant allele. Left to right: lane 1 is 100-base pair (bp) DNA ladder and lanes 2, 3, 5, and 6 are JAK2 p. Val617Phe positive while lanes 4 and 7 are wild type cases

Iranian Ph-negative MPNs and did not detect any $M P L$ mutations. In contrast, ElNahass et al. screened 93 classical Egyptian MPN patients for the detection of different $M P L$ pathological variants and detected a frequency of 3\% among ET and PMF patients [19]. The authors screened all the MPL mutations by using a high-resolution melting analysis (HRM) [19]. Also, Lieu et al. [17] detected higher frequency of MPL p. W515 L/K among ET and PMF compared to that among PV. In this study, 14/60 (23\%) of our patients were with PV. Furthermore, the frequency of $M P L$ p. W $515 \mathrm{~L} / \mathrm{K}$ is higher in JAK2-negative MPN [20]. About $50 \%$ of our patients were positive for $J A K 2$ p. Val617Phe pathological variant. Screening for $M P L \mathrm{p}$. W $515 \mathrm{~L} / \mathrm{K}$ is recommended for the JAK2 p. Val617Phe-negative MPN [21, 22].

The prevalence of MPL mutations in MPN patients varies across different ethnic groups. In China, Lin et al. [13] studied 929 Chinese patients and detected $M P L$ mutations in $1.2 \%$ in ET and $2.7 \%$ in PMF. Xu et al. [23] screened $190 \mathrm{MPN}$ patients and found that $M P L \mathrm{p} . \mathrm{W} 15 \mathrm{~L} / \mathrm{K}$ pathological variants were detected in $1 \%$ of ET patients, and none was found in PMF patients. In Europe, Usseglio et al. (2016) found only $3 \%$ of ET and $1 \%$ of PMF patients positive for MPL p. W515 L/K pathological variants among $164 \mathrm{MPN}$ patients from France [24] while Rumi et al. screened 617 PMF patients from Italy \& Spain and detected $4.1 \%$ of patients are positive for $M P L \mathrm{p} . \mathrm{W} 15 \mathrm{~L} / \mathrm{K}$ pathological variants [25]. In Poland, Wojtaszewska et al. screened 184 Polish patients who were JAk2 p. Val617Phe pathological variant-negative MPNs and only $1 \%$ of ET patients had MPL mutation while none of the PMF was positive for the pathological variant [26]. Going to the Middle East, Akpinar et al. screened Turkish MPN patients and found $M P L$ p. W $515 \mathrm{~L} / \mathrm{K}$ pathological variants with a frequency of $2.6 \%$ across PMF patients while they did not find any in ET patients [27]. In Iran, Shams et al. screened $105 \mathrm{MPN}$ patients and the frequency was $4.76 \%$ [28].

In this study, our MPN patients showed a much lower median age at diagnosis of 49.5, 45, and 56.5 in PV, ET, and PMF, respectively, compared with other studies which had a higher median age of 59,55 , and 65 in PV, ET, and PMF, respectively [29-31]. This study revealed lower frequency of Jak2 p. Val617Phe pathological variant across PV and PMF represented in 64.3 and $30 \%$, respectively, when compared to other data worldwide for the incidence of Jak2 p. Val617Phe pathological variant which was represented in 85 and $65.8 \%$ [13], 81.4 and $46.1 \%$ [32], 87.2 and $50 \%$ [2], $100 \%$ and 68\% [33], and 90 and $62 \%$ [19] of PV and PMF, respectively.

The percentage of ET patients who had splenomegaly at diagnosis was $50 \%$ which is considered to be higher than the percentage of other ET patients worldwide (5-20\%) [34]. This deviation may be related to endemic parasitic infections like schistosomiasis in Egyptian patients. Also, our ET patients were considered relatively young and the incidence of splenomegaly we reported was high in young patients which is consistent with the results reported by Montanaro et al. [34].

\section{Conclusion}

The current study did not detect any MPL p. W515 $\mathrm{L} / \mathrm{K}$ pathological variants in the Egyptian population. The frequency of JAK2 p. Val617Phe pathological variant in PV and PMF patients was lower 
Table 3 Initial patients' characteristics for PV, ET, and PMF

\begin{tabular}{|c|c|c|c|c|}
\hline Variables (clinical and laboratory findings) & $\begin{array}{l}\text { Polycythemia Vera (PV) } \\
N=14\end{array}$ & $\begin{array}{l}\text { Essential thrombocythemia (ET) } \\
N=26\end{array}$ & $\begin{array}{l}\text { Primary myelofibrosis (PMF) } \\
N=20\end{array}$ & $P$ value \\
\hline Female, no. (\%) & $6(42.9)$ & $13(50)$ & $7(35.0)$ & 0.597 \\
\hline Male, no. (\%) & $8(57.1)$ & $13(50)$ & $13(65.0)$ & \\
\hline \multicolumn{5}{|l|}{ Age } \\
\hline Mean \pm SD & $47 \pm 13.6$ & $47 \pm 14$ & $53.6 \pm 12.6$ & 0.236 \\
\hline Median & 49.5 & 45 & 56.5 & \\
\hline Min-max & $22-73$ & $26-71$ & $24-73$ & \\
\hline \multicolumn{5}{|l|}{ WBC $\left(10^{9} / L\right)$} \\
\hline Mean \pm SD & $8.8 \pm 3.3$ & $11.8 \pm 9.9$ & $11.9 \pm 15.9$ & 0.679 \\
\hline Median & 8 & 8 & 6.4 & \\
\hline Min-max & $4.5-17$ & $5-50$ & $1-69$ & \\
\hline \multicolumn{5}{|l|}{ Hemoglobin (g/dL) } \\
\hline Mean \pm SD & $17.4 \pm 2.4$ & $13 \pm 3.5$ & $8.7 \pm 2.8$ & $<0.001$ \\
\hline Median & 17.7 & 13 & 8.5 & \\
\hline Min-max & $12.3-21$ & $8-26$ & $3-14.9$ & \\
\hline \multicolumn{5}{|l|}{ Platelet count $\left(10^{9} / \mathrm{L}\right)$} \\
\hline Mean \pm SD & $294 \pm 165$ & $1099 \pm 470$ & $268.5 \pm 351.8$ & $<0.001$ \\
\hline Median & 347.5 & 922 & 150.5 & \\
\hline Min-max & $32-570$ & $455-2433$ & $13-1408$ & \\
\hline JAK2 p. Val617Phe & $9(64.3)$ & $13(50)$ & $6(30)$ & 0.143 \\
\hline \multicolumn{5}{|l|}{ Splenomegaly, no. (\%) } \\
\hline Positive & $3(21.4)$ & $13(50)$ & $11(55.0)$ & 0.126 \\
\hline Negative & $11(78.6)$ & $13(50)$ & $9(45)$ & \\
\hline \multicolumn{5}{|l|}{ Hepatomegaly, no. (\%) } \\
\hline Positive & $1(7.1)$ & $4(15.4)$ & $4(20.0)$ & 0.527 \\
\hline Negative & $13(92.9)$ & $22(84.6)$ & $16(80)$ & \\
\hline \multicolumn{5}{|l|}{ Fatigue, no. (\%) } \\
\hline Positive & $5(35.7)$ & $6(23.1)$ & $12(60)$ & 0.041 \\
\hline Negative & $9(64.3)$ & $20(76.9)$ & $8(40)$ & \\
\hline \multicolumn{5}{|l|}{ Headache, no. (\%) } \\
\hline Positive & $4(28.6)$ & $7(26.9)$ & $5(25)$ & 1.000 \\
\hline Negative & $10(71.4)$ & $19(73.1)$ & $15(75)$ & \\
\hline \multicolumn{5}{|l|}{ Bleeding events, no. (\%) } \\
\hline Positive & $1(7.1)$ & $5(19.2)$ & $5(25)$ & 0.443 \\
\hline Negative & $13(92.9)$ & $21(80.8)$ & $15(75)$ & \\
\hline \multicolumn{5}{|l|}{ Thrombosis, no. (\%) } \\
\hline Positive & $1(7.1)$ & $4(15.4)$ & $1(5)$ & 0.551 \\
\hline Negative & $13(92.9)$ & $22(84.6)$ & $19(95)$ & \\
\hline \multicolumn{5}{|l|}{ Dizziness, no. (\%) } \\
\hline Positive & $0(0)$ & $2(7.7)$ & $4(20)$ & 0.180 \\
\hline Negative & $14(100)$ & $24(92.3)$ & $16(80)$ & \\
\hline
\end{tabular}

WBC white blood cells, SD standard deviation 
than other studies. MPNs affect Egyptians at a younger age compared to different ethnic groups. There is a high frequency of splenomegaly among Egyptian ET patients compared to other studies over different populations. We recommend further studies on a larger number of patients with MPNs in Egypt.

\begin{abstract}
Abbreviations
MPNs: Myeloproliferative neoplasmsPh-negativePhiladelphianegativeARMSAmplification refractory mutation systemPVPolycythemia veraETEssential thrombocythemiaPMFPrimary myelofibrosisCMLChronic myelogenous leukemiaWHOWorld Health OrganizationTPOThrombopoietin receptorWBCWhite blood cellsSDStandard deviationHRMHigh-resolution melting analysis
\end{abstract}

Acknowledgements

Not applicable.

\section{Authors' contributions}

GE conceived and planned the experiments. SE carried out the experiments. $\mathrm{GE}, \mathrm{HI}$, and MS helped supervise the project. SE wrote the manuscript with support from GE, HI, and MS. SE, GE, HI, and MS have contributed in the data analysis and interpretation. $\mathrm{SE}, \mathrm{GE}, \mathrm{HI}$, and MS have agreed both to be personally accountable for the author's own contributions and to ensure that questions related to the accuracy or integrity of any part of the work, even ones in which the author was not personally involved, are appropriately investigated, resolved, and the resolution documented in the literature. All authors have read and approved the submitted manuscript version.

\section{Funding}

This research did not receive any specific grant from funding agencies in the public, commercial, or not-for-profit sectors.

\section{Availability of data and materials}

The datasets used and/or analyzed during the current study are available from the corresponding author on reasonable request.

\section{Ethics approval and consent to participate}

The collected data is de-identified for a retrospective study, and the ethics approval is unnecessary according to the Research ethics

Committee-MoHP.

\section{Consent for publication}

Not applicable.

\section{Competing interests}

The authors declare that they have no competing interests.

\section{Author details}

'Department of Chemistry, Faculty of Science, Cairo University, Al Gamaa street, Al Orman, Giza 12613, Egypt. ${ }^{2}$ Department of Clinical Pathology \& Oncologic Laboratory Medicine, National Cancer Institute, Cairo University, Kasr Al Eini Street, Fom El Khalig, Cairo 11796, Egypt. ${ }^{3}$ Department of Medical Oncology \& Hematology, National Cancer Institute, Cairo University, Kasr Al Eini Street, Fom El Khalig, Cairo 11796, Egypt.

\section{Received: 5 August 2019 Accepted: 4 November 2019}

\section{Published online: 12 December 2019}

\section{References}

1. Skoda RC, Duek A, Grisouard J (2015) Pathogenesis of myeloproliferative neoplasms. Exp Hematol. 43(8):599-608. https://doi.org/10.1016/j.exphem. 2015.06.007

2. Azevedo AP, Silva S, Reichert A, Lima F, Junior E, Rueff J (2017) Prevalence of the Janus kinase 2 V617F mutation in Philadelphia-negative myeloproliferative neoplasms in a Portuguese population. Biomed Reports.: 370-376. https://doi.org/10.3892/br.2017.977
3. Arber DA, Orazi A, Hasserjian R, Thiele J, Borowitz MJ, Le Beau MM et al (2016) The 2016 revision to the World Health Organization classification of myeloid neoplasms and acute leukemia. Blood. 127(20):2391-2406. https:// doi.org/10.1182/blood-2016-03-643544

4. Boddu P, Chihara D, Masarova L, Pemmaraju N, Patel KP, Verstovsek S (2018) The co-occurrence of driver mutations in chronic myeloproliferative neoplasms. Ann Hematol. https://doi.org/10.1007/s00277-018-3402-x

5. Xia J, Lu MZ, Jiang YQ, Yang GH, Zhuang Y, Sun HL et al (2012) JAK2 V617F, MPL W515 L and JAK2 exon 12 mutations in Chinese patients with primary myelofibrosis. Chinese J Cancer Res. 24(1):72-76. https://doi.org/10.1007/ s11670-012-0072-4

6. Leszczynska A, Grzenkowicz-Wydra J, Chmielewska-Gorycka L, Bieniaszewska M, Hellmann A (2016) Detection of JAK2 Exon 12 mutations in JAK2 V617Fnegative polycythemia vera. Acta Haematol (exon 14):123-128. https://doi. org/10.1159/000446798

7. Tefferi A, Lasho T, Finke C, Knudson RA, Ketterling R, Hanson CH et al (2014) CALR vs JAK2 vs MPL-mutated or triple-negative myelofibrosis: clinical, cytogenetic and molecular comparisons. Leukemia. 28(7):1472-1477. https:// doi.org/10.1038/leu.2014.3

8. Ji L, Qian M, Wu N, Wu J (2017) Significance of combined detection of JAK2V617F, MPL and CALR gene mutations in patients with essential thrombocythemia. Exp Ther Med. 13(3):947-951. https://doi.org/10.3892/ etm.2017.4077

9. O'Sullivan JM, Harrison CN (2017) JAK-STAT signaling in the therapeutic landscape of myeloproliferative neoplasms. Mol Cell Endocrinol. 451:71-79. https://doi.org/10.1016/j.mce.2017.01.050

10. Arunachalam AK, Suresh $H$, Mathews V, Balasubramanian P (2018) Allele specific PCR : a cost effective screening method for MPL mutations in myeloproliferative neoplasms. Indian J Hematol Blood Transfus:1543-1545. https://doi.org/10.1007/s12288-018-0982-5

11. Lasho TL, Pardanani A, McClure RF, Mesa RA, Levine RL, Gilliland DG et al (2006) Concurrent MPL515 and JAK2V617F mutations in myelofibrosis: chronology of clonal emergence and changes in mutant allele burden over time. Br J Haematol. 135(5):683-687. https://doi.org/10.1111/j.1365-2141. 2006.06348.x

12. Staerk J, Defour JP, Pecquet C, Leroy E, Antoine-Poirel H, Brett I et al (2011) Orientation-specific signalling by thrombopoietin receptor dimers. EMBO J. 30(21):4398-4413. https://doi.org/10.1038/emboj.2011.315

13. Lin Y, Liu E, Sun Q, Ma J, Li Q, Cao Z et al (2015 Jul) The Prevalence of JAK2, MPL, and CALR mutations in Chinese patients with BCR-ABL1-negative myeloproliferative neoplasms. Am J Clin Pathol. 144(1):165-171. https://doi. org/10.1309/AJCPALP51XDIXDDV

14. Langabeer SE, Andrikovics H, Asp J, Bellosillo B, Carillo S, Haslam K et al (2015) Molecular diagnostics of myeloproliferative neoplasms. Eur J Haematol. 95(4):270-279. https://doi.org/10.1111/ejh.12578

15. Zhuge J, Zhang W, Xu M, Hoffman R, Zhang W (2010) Sensitive detection of MPLW515 L/K mutations by amplification refractory mutation system (ARMS)PCR. Clin Chim Acta. 411(1-2):122-123. https://doi.org/10.1016/j.cca.2009.10.012

16. Chen Q, Lu F, Jones AV, Cross NC, Silver RT, Wang YL (2007) Amplification refractory mutation system, a highly sensitive and simple polymerase chain reaction assay, for the detection of JAK2 V617F mutation in chronic myeloproliferative disorders. J Mol Diagn. 9(2):272-276. https://doi.org/10. 2353/jmoldx.2007.060133

17. Lieu CH, Shen YJ, Lai WC, Tsai WH, Hsu HC (2010) Prevalence of MPL W515 $\mathrm{L} / \mathrm{K}$ Mutations in Taiwanese patients with Philadelphia-negative chronic myeloproliferative neoplasms. J Chinese Med Assoc. 73(10):530-532. https:// doi.org/10.1016/S1726-4901(10)70115-5

18. Ghotaslou A, Nadali F, Chahardouli B, Alizad Ghandforosh N, Rostami SH, Alimoghaddam K et al (2015) Low frequency of c-MPL gene mutations in Iranian patients with Philadelphia-negative myeloproliferative disorders. Iran J Ped Hematol Oncol. 5(1):43-49

19. EINahass YH, Mahmoud HK, Mattar MM, Fahmy OA, Samra MA, Abdelfattah RM et al (2018) MPN10 score and survival of molecularly annotated myeloproliferative neoplasm patients. Leuk Lymphoma. 59(4):844-854. https://doi.org/10.1080/10428194.2017.1365852

20. Guglielmelli P, Pancrazzi A, Bergamaschi G, Rosti V, Villani L, Antonioli E et al (2007) Anaemia characterises patients with myelofibrosis harbouring MplW515 L/Kmutation. Br J Haematol. 137(3):244-247. https://doi.org/10. 1111/j.1365-2141.2007.06565.x

21. Ruan G-R, Jiang B, Li L-D, Niu J-H, Li J-L, Xie M et al (2009) MPL W515 L/K mutations in 343 Chinese adults with JAK2V617F mutation-negative chronic 
myeloproliferative disorders detected by a newly developed. Hematol Oncol. 25(3):127-131. https://doi.org/10.1002/hon

22. Furtado LV, Weigelin HC, Elenitoba-Johnson KS, Betz BL (2013) Detection of MPL mutations by a novel allele-specific PCR-based strategy. J Mol Diagnostics. 15(6):810-818. https://doi.org/10.1016/j.jmoldx.2013.07.006

23. Xu W, Li JY, Xia J, Zhang SJ, Fan L, Qiao C (2008) MPL W515 L mutation in Chinese patients with myeloproliferative diseases. Leuk Lymphoma. 49(May): 955-958. https://doi.org/10.1080/10428190802035966

24. Usseglio F, Beaufils N, Calleja A, Raynaud S, Gabert J (2017) Detection of CALR and MPL mutations in low allelic burden JAK2 V617F essential thrombocythemia. J Mol Diagnostics. 19(1):92-98. https://doi.org/10.1016/j. jmoldx.2016.08.006

25. Rumi E, Pietra D, Pascutto C, Guglielmelli P, Martínez-Trillos A, Casetti I et al (2014) Clinical effect of driver mutations of JAK2, CALR or MPL in primary myelofibrosis key points. Blood. https://doi.org/10.1182/blood-2014-05-578435

26. Wojtaszewska M, Małgorzata I, Lewandowski K (2015) Frequency and molecular characteristics of Calreticulin Gene (CALR) mutations in patients with JAK2-negative myeloproliferative neoplasms. Acta Haematol. 133(2): 193-198. https://doi.org/10.1159/000366263

27. Akpınar TS, Hançer VS, Nalçacı M (2013) Diz- Küçükkaya R. MPL W515 L/K mutations in chronic myeloproliferative neoplasms. Turkish J Hematol. 30(1): 8-12. https://doi.org/10.4274/tjh.65807

28. Shams SF, Ayatollahi H, Sadeghian MH, Afzalaghaee M, Shakeri S, Yazdandoust E et al (2018) Prevalence of MPL (W515K/L) mutations in patients with negative-JAK2 (V617F) myeloproliferative neoplasm in NorthEast of Iran. Iran J Pathol. 13(4):397-402

29. Passamonti F, Rumi E, Pungolino E, Malabarba L, Bertazzoni P, Valentini M et al (2004) Life expectancy and prognostic factors for survival in patients with polycythemia vera and essential thrombocythemia. Am J Med 117(10): 755-761. https://doi.org/10.1016/j.amjmed.2004.06.032

30. Cervantes F, Dupriez B, Pereira A, Passamonti F, Reilly JT, Morra E et al (2009) New prognostic scoring system for primary myelofibrosis based on a study of the International Working Group for Myelofibrosis Research and Treatment. Blood. 113(13):2895-2901. https://doi.org/10.1182/blood-2008-07-170449

31. Song MK, Park BB, Uhm JE (2018) Understanding splenomegaly in myelofibrosis: association with molecular pathogenesis. Int J Mol Sci 19(3). https://doi.org/10.3390/ijms19030898

32. Ayad MW, Nafea D (2011) Acquired mutation of the tyrosine kinase JAK2V617F in Egyptian patients with myeloid disorders. 15(1):17-21. https:// doi.org/10.1089/gtmb.2010.0093

33. Cacemiro MDC, Cominal JG, Tognon R, Nunes NS, Simões BP, FiqueiredoPontes LL et al (2018) Philadelphia-negative myeloproliferative neoplasms as disorders marked by cytokine modulation. Hematol Transfus Cell Ther. 40(2): 120-131. https://doi.org/10.1016/j.htct.2017.12.003

34. Andriani A, Latagliata R, Anaclerico B, Spadea A, Rago A, Di Veroli A et al (2016) Spleen enlargement is a risk factor for thrombosis in essential thrombocythemia: evaluation on 1,297 patients. Am J Hematol. 91(3):318321. https://doi.org/10.1002/ajh.24269

\section{Publisher's Note}

Springer Nature remains neutral with regard to jurisdictional claims in published maps and institutional affiliations.

\section{Submit your manuscript to a SpringerOpen ${ }^{\circ}$ journal and benefit from:}

- Convenient online submission

- Rigorous peer review

- Open access: articles freely available online

- High visibility within the field

- Retaining the copyright to your article

Submit your next manuscript at $\boldsymbol{\nabla}$ springeropen.com 